\title{
基于 $\mathrm{MoS}_{2}-\mathrm{XS}(\mathrm{X}=\mathrm{Al}, \mathrm{B}, \mathrm{Ga})$ 复合结构光能的高效利用
}

\author{
李泓霖 ${ }^{*}$, 熊元强, 张红, 叶利娟, 李万俊 ${ }^{*}$ \\ 重庆师范大学物理与电子工程学院, 重庆市光电功能材料重点实验室, 重庆 401331 \\ *联系人, E-mail: 20170025@cqnu.edu.cn; liwj@cqnu.edu.cn
}

2021-03-28 收稿, 2021-05-12 修回, 2021-05-17 接受, 2021-05-18 网络版发表

国家自然科学基金(11904041)、重庆市自然科学基金(cstc2020jcyj-msxmX0557, cstc2020jcyj-msxmX0533)和重庆市教育委员会科学技术研究 项目(KJQN20200051，KJQN201900542)资助

\begin{abstract}
摘要 近年来, 二维过渡金属硫化物材料因其独特的物化属性受到了广泛的关注, 并已成功应用于微电子器件、 光伏技术及析氢催化等诸多领域, 展示出了广阔的应用前景. 然而, 高载流子复合率、低迁移率和低循环稳定性等 问题都在不同程度上限制了该类材料相关性能的进一步提升. 目前, 将二维材料复合处理, 对能带结构或物化属性 等进行优化改进, 在诸多实验中已被证实是切实可行的. 基于密度泛函理论, 本研究提出了 $\mathrm{MoS}_{2}-\mathrm{XS}(\mathrm{X}=\mathrm{Al}, \mathrm{B}, \mathrm{Ga})$ 异质结构. 研究发现, $\mathrm{MoS}_{2}$ - $\mathrm{AlS} / \mathrm{BS}$ 复合结构具有极低的失配度和天然的II型异质结能带形式; $\mathrm{MoS}_{2}-\mathrm{XS}$ 的价带顶和 导带底分别由 $\mathrm{MoS}_{2}$ 和XS构成, 但异质结构具有比纯净结构更低的有效质量和更好的输运特性; 当 $\mathrm{MoS}_{2}-\mathrm{BS}$ 复合结 构应用于太阳能电池时, 其能量转换效率高达 20.4\%.
\end{abstract}

关键词 $\mathrm{MoS}_{2}$, 二维材料, III-VI族化合物, 异质结, 第一性原理

自石墨烯成功剥离以来，二维材料以其丰富多样 的物化属性多年来一直是纳米材料和纳米器件研究中 最有应用前景的方向之一 ${ }^{[1 ~ 3]}$. 其中，二维过渡金属硫 化物(transition metal dichalcogenides, TMDCs)是重要 的组成部分, 而 $\mathrm{MoS}_{2}$ 又是该体系中最为广泛深人研究 的材料. TMDCs层内原子通过稳定的共价键连结, 不 同层间以弱范德华力(van der Waals force, vdW)相结 合，这使得二维层状材料的高效制备和广泛应用成为 可能. 大多数 TMDCs (如 $\mathrm{WS}_{2} / \mathrm{MoSe}_{2}$ )从体结构剥离成 单层时, 其带隙将由间接带隙逐渐过渡到直接带隙 $[4,5]$. 这种结构特征和电子属性使得基于TMDCs的二维材料 在光电子器件 ${ }^{[6,7]}$ 、光电催化 ${ }^{[8]}$ 、新能源开发 ${ }^{[9,10]}$ 和自 旋电子学 ${ }^{[11,12]}$ 等前沿领域中展现了巨大潜力. 单层 TMDCs在可见光范围内具有良好的吸收能力、高开关 比和良好的化学稳定性 ${ }^{[13 \sim 15]}$. 然而, 其较低的迁移率、 易受环境影响的结构特性和显著的载流子复合现象在
一定程度上影响了该类材料的进一步应用 ${ }^{[16]}$. 为了合 理可行地解决上述困境, 必须通过某些方法进行优化 改进, 其中一个可能的途径是通过复合处理实现II型异 质结的制备 ${ }^{[17]}$. 一般而言, 复合结构中材料的不同部分 对能带的贡献有所区别，电子/空穴也会由于费米能级 的不同而位于不同的区域，从而自发形成的内建电场 可以更好地促使光生载流子得到分离. 然而, 理想异质 结构的设计并非易事, 这是因为所选的材料必须满足 两个前提条件: 一是价带顶(valence band maximum, $\mathrm{VBM}$ )和导带底(conduction band minimum, CBM)应分 属复合材料的不同部分, 以实现光生载流子的高效分 离; 二是复合结构的能带边缘应位于恰当位置以保证 适于特定的应用 ${ }^{[18,19]}$.

Zhou等人 ${ }^{[20]}$ 构建了 $\mathrm{C}_{2} \mathrm{~N} /$ BlueP vdW异质结构, 计算 研究了相应的电子特性和能带排列等, 分析了其复合 结构层间距改变后光催化性能的变化趋势. 结果表明, 
$\mathrm{C}_{2} \mathrm{~N} / \mathrm{BlueP}$ 复合结构的直接带隙和本征II型能带排列都 非常利于光生电子-空穴对的分离转移, 表明 $\mathrm{C}_{2} \mathrm{~N} / \mathrm{BlueP}$ 复合结构有一定的光催化应用潜力. 对复合结构施加 一定的垂直压力, 层间距从最稳定的 $3.354 \AA$ 减小到 $3.154 \AA$ 后, 其能带排布得到了进一步的改善. 低能部 分的光吸收得到了一定程度的增强, 同时电子和空穴 的迁移率分别提高了 $25 \%$ 和 $130 \%$, 体现了更好的光催 化效率. $\mathrm{Vu}$ 等人 ${ }^{[21]}$ 构建了石墨烯/WSeTe复合结构, 并 计算研究了其结构属性、光学性质和载流子传输特征. 研究发现, 与石墨烯和Janus WSeTe单层结构相比, 复 合结构的光吸收在可见光区和紫外光区中均得到了增 强. 复合结构可见光的吸收系数高达 $5 \times 10^{4} \mathrm{~cm}^{-1}$, 是单 层Janus WSeTe的两倍. 计算结果表明, 电场可以有效 地用于调控复合结构的接触类型和肖特基势垒高度, 使其从 $\mathrm{p}$ 型肖特基接触转变到 $\mathrm{n}$ 型接触或 $\mathrm{p}$ 型欧姆接触. 此外, 由于二维材料具有较高的结构强度, 施加外应变 被认为是调节能带排列并改进界面性能的有效方法. Liu等人 ${ }^{[22]}$ 从实验上提出了一种利用一维半导体纳米 压痕构造周期性应变 $\mathrm{vdWs}$ 异质结构阵列的方法. 其复 合体系为单层 $\mathrm{MoS}_{2} / \mathrm{ZnO}$ 阵列, 且异质结构的应变范围 在 $0 \sim 0.6 \%$. 光学表征发现, 应变确实可以提高界面处的 电荷转移效率, 受应变作用的单层 $\mathrm{MoS}_{2}$ 在应变界面处 的光致发光强度大幅低于非应变界面 $50 \%$ 以上. 综上, 改变层间距、施加外电场和外应变均会对复合结构产 生一定的影响, 这正是我们着重研究这3种外部作用的 原因所在.

2017年, Demirci等人 ${ }^{[23]}$ 研究了III-VI族二维单层六 角结构 $\mathrm{MX}(\mathrm{M}=\mathrm{B}, \mathrm{Al}, \mathrm{Ga}, \mathrm{In} ; \mathrm{X}=\mathrm{O}, \mathrm{S}, \mathrm{Se}, \mathrm{Te})$. 一系列 分析表明, 上述所有二元化合物都是结构稳定的. 他们 认为, III-VI族二元单层材料可广泛地适用于高性能光 电器件. 基于此, 我们将对 MX作进一步的异质复合研 究. $\mathrm{MoS}_{2}$ 与 $\mathrm{MS}(\mathrm{M}=\mathrm{B}, \mathrm{Al}, \mathrm{Ga})$ 具有相同的六方晶体结 构(P-6m2), 有理由相信在一定的实验条件下会合成出 $\mathrm{MoS}_{2}-\mathrm{XS}$ 异质复合结构. 因此, 了解单层 $\mathrm{MoS}_{2}$ 和XS如 何形成稳定的二维异质结构以及相关的潜在应用是十 分有意义的. 目前, $\mathrm{MoS}_{2}-\mathrm{XS}$ 的结构性质和相关物化属 性未见报道, 因此本研究将对 $\mathrm{MoS}_{2}$-XS复合结构的基 本特性及潜在实际应用进行探究.

\section{1 计算结果与讨论}

基于二维结构周期性边界条件, 复合结构的建立 需首先严格控制 $\mathrm{MoS}_{2}$ 和XS之间的失配度, 相关计算流
程与细节详见补充材料. 在研究复合材料的电子结构 之前, 需确定出单层结构的电子特性. 图1(a)为单层 $\mathrm{MoS}_{2}$ 的能带结构. $\mathrm{MoS}_{2}$ 是由 3 层原子组成的稳定六方 晶体, 其晶格常数为 $a=b=3.25 \AA . \mathrm{S}$ 态贡献了 $\mathrm{MoS}_{2}$ 的 VBM和CBM, 而Mo原子态主要位于价带的深能级处, 对材料的整体性能影响很小, 其带隙约为 $1.74 \mathrm{eV}$, 与之 前文献[24]报道结果一致. 图1(b)所示AlS的能带结构 与 $\mathrm{MoS}_{2}$ 明显不同. $\mathrm{AlS}$ 的带隙为 $2.12 \mathrm{eV}$, 显著大于 $\mathrm{MoS}_{2}$. BS和 $\mathrm{GaS}$ 的间隙分别为 2.90 和 $2.39 \mathrm{eV}$. 一般而 言, 减小带隙可以在一定程度上提高光能的利用率, 这 也是掺杂技术的目的之一. 然而, 掺杂会不可避免地引 人外来原子作为复合中心, 并增强光生载流子的复合, 因此, 对材料进行复合处理是一个解决载流子强复合 的可行方案. 当半导体被光照射时, 电子将被激发到导 带(conduction band, CB)并在价带(valence band, VB)中 留下空穴. 一部分载流子会自发复合, 不再参与后续反 应, 另一部分则会迅速迁移到外层空间, 并参与随后的 物化反应. 理想的情况是第一部分中载流子的复合尽 可能受到抑制并尽可能多地参与后续反应.

一般而言, 光解水过程中激子在催化剂和水分子 之间的输运是关键环节, 光生电子/空穴只有在遵循热 力学定律的前提下才能作用于相关反应 ${ }^{[25]}$. 具体而言, 标准氢电极 (normal hydrogen electrode, NHE)通常用于 电化学研究, 同时也是水解氧化还原标准电势, 由氧化 电势和还原电势组成. 在 $\mathrm{pH} 0$ 和 $25^{\circ} \mathrm{C}$ 条件下, 氧化电 位和还原电位相对于真空能级分别约为 -5.67 和 $-4.44 \mathrm{~V}^{[26]}$. 实际反应中, 催化剂的能带边缘位置是决 定水解反应是否可行的重要参数. 理想情况下, 可自发 进行光水解反应半导体的 VBM应比氧化电位 $\mathrm{O}_{2} / \mathrm{H}_{2} \mathrm{O}$ 更高, 且 $C B M$ 比还原电位 $\mathrm{H}^{+} / \mathrm{H}_{2}$ 更低 ${ }^{[27,28]}$. 本研究中, $\mathrm{VBM}$ 在数值上等于半导体相对于真空能级的功函数 $(\varphi): \varphi=V(\infty)-E_{\mathrm{f}}$. 在2D模型中, $V(\infty)$ 表示远离界面的静 电势, 约等于真空能级. 因此, 后续计算出的能带边缘 可对应于实验中的氧化还原电位. 从图1(a) (d)可以发 现, 4种单层结构都是理想的光解水催化剂, 其VBM和 $\mathrm{CBM}$ 都分别远离 $\mathrm{O}_{2} / \mathrm{H}_{2} \mathrm{O}$ 和 $\mathrm{H}^{+} / \mathrm{H}_{2}$, 体现了相当的氧化还 原能力. 值得注意的是, 4 种材料的 $\mathrm{VBM}$ 和 CBM 都不同 程度地由 $\mathrm{S}$ 原子态构成, 尤其是 $\mathrm{MoS}_{2}$ 和AlS, 其能带边 缘几乎都来自 $\mathrm{S}$ 原子. 这将大幅提高载流子的复合, 使 得水解反应中能够实际利用的电子和空穴显著减少. 因此, 如何抑制载流子的复合将是本文重要的研究目 的之一。 

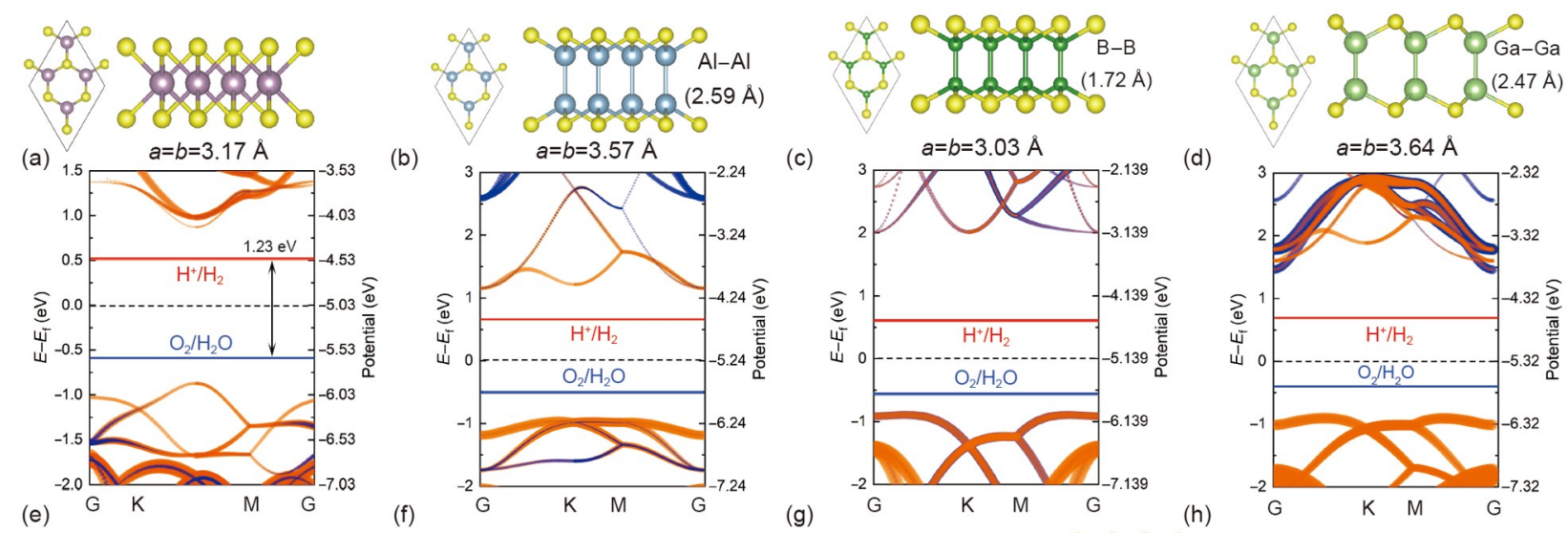

(c)

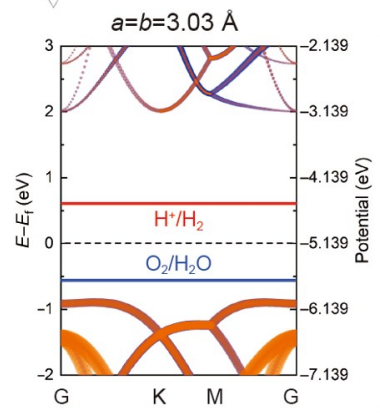

(d)
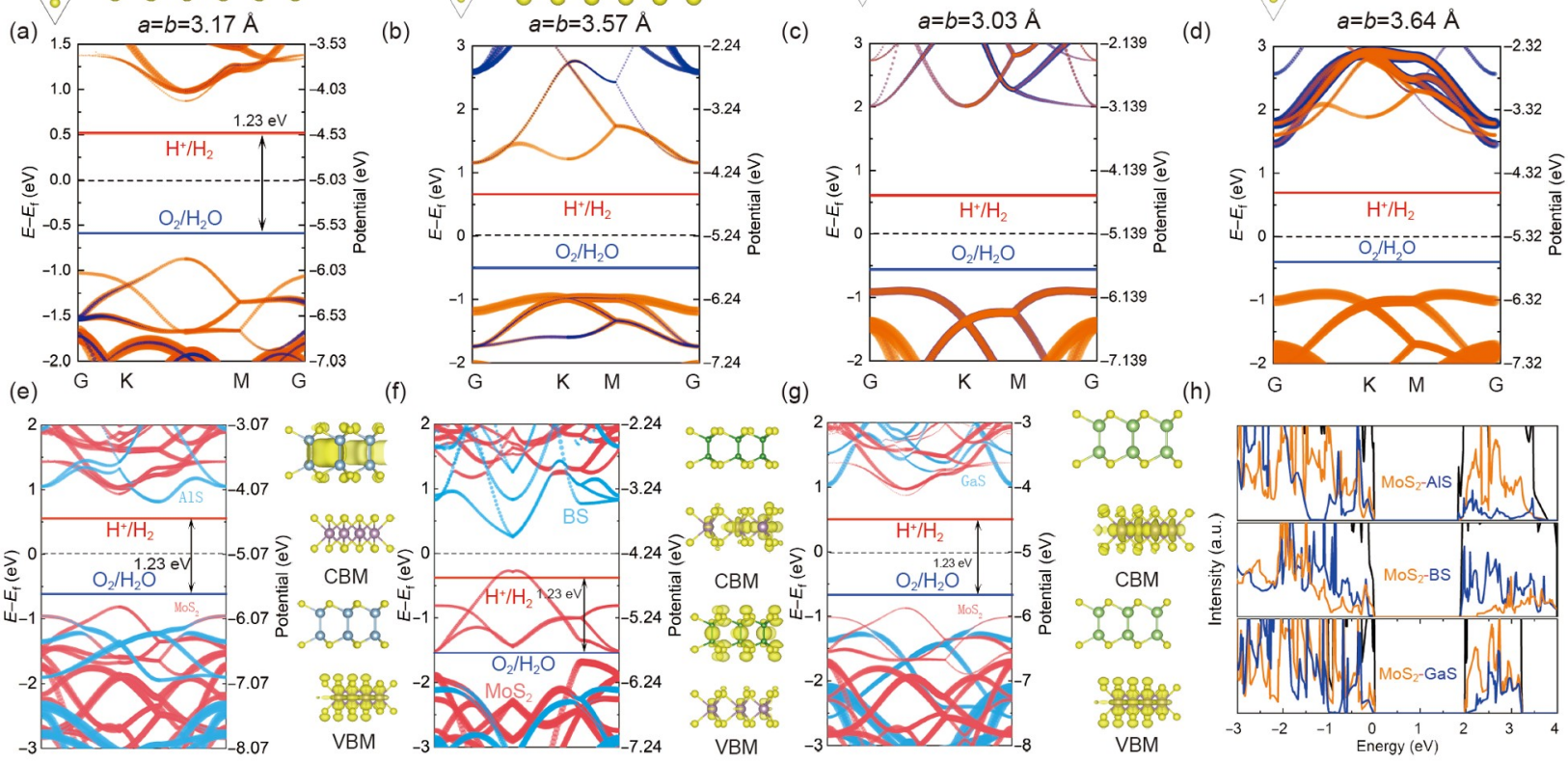

图 1 不同结构的原子示意图及相应的能带结构. (a) $\mathrm{MoS}_{2}$; (b) AlS; (c) BS; (d) GaS. MoS $-\mathrm{AlS}(\mathrm{e}) 、 \mathrm{MoS}_{2}-\mathrm{BS}(\mathrm{f})$ 和MoS $2-\mathrm{GaS}(\mathrm{g})$ 的投影能带图, 右 侧为相应的VBM/CBM电荷密度图. (h) 3 种复合结构的分态密度图

Figure 1 Schematic diagrams of atoms with different structures and the corresponding band structures. (a) MoS 2 ; (b) AlS; (c) BS; (d) GaS. The projected band structures of $\mathrm{MoS}_{2}-\mathrm{AlS}(\mathrm{e}), \mathrm{MoS}_{2}-\mathrm{BS}(\mathrm{f})$ and $\mathrm{MoS}_{2}-\mathrm{GaS}(\mathrm{g})$. The right figures show the decomposed charge density of the VBM/CBM. (h) Partial density of states of three heterostructures

图1(e) (h)是 $\mathrm{MoS}_{2}-\mathrm{XS}$ 复合结构的原子投影能带图 和分态密度图，线条的粗细渐变代表相应成分贡献的 权重大小. 相关的 $\mathrm{MoS}_{2}-\mathrm{XS}$ 复合结构晶格参数在表 $\mathrm{S} 1$ 中给出. 显然, $\mathrm{MoS}_{2}-\mathrm{AlS}$ 和MoS 2 -BS复合结构都形成了 典型的II型异质结能带排列，因而载流子可被高效分 离. 这两个复合结构中的VBM完全由 $\mathrm{MoS}_{2}$ 贡献，而 CBM来自AlS/BS. 进一步观察发现, $\mathrm{MoS}_{2}$-AlS的带隙 下降至 $1.62 \mathrm{eV}$, 显然比纯净 $\mathrm{MoS}_{2}$ 和AlS的带隙小. 这种 特殊的能带排列形式可自发地分离光生电子-空穴对, 有利于高效光电器件的制备 ${ }^{[29,30]}$. 自由状态下, $\mathrm{MoS}_{2}$ 和 AlS/BS都可在吸收光子后将VB中的电子激发到 $\mathrm{CB}$ 并 在VB中留下空穴. 而后, $\mathrm{MoS}_{2}$ 中的光生电子将在电势 作用下注人 $\mathrm{AlS} / \mathrm{BS}$ 的CBM中，同时光生空穴将迅速积 聚到 $\mathrm{MoS}_{2}$ 的VBM. 图1(f), (g)中 $\mathrm{MoS}_{2}-\mathrm{BS}$ 和 $\mathrm{MoS}_{2}-\mathrm{GaS}$ 带隙分别减小至 0.63 和 $1.92 \mathrm{eV}$ ，也都小于纯净结构的 带隙. 需要说明的是, $\mathrm{MoS}_{2}-\mathrm{GaS}$ 复合结构为本征I型异 质结, 其CBM和VBM均由 $\mathrm{MoS}_{2}$ 贡献. 总之, II型异质结 的载流子分离特性对高性能光催化剂或光伏电池的制 造十分有利 ${ }^{[31,32]}$.
为了定性说明 $\mathrm{MoS}_{2}-\mathrm{XS}$ 内部电荷转移情况, 我们 计算了 3 种复合结构的电荷密度差(charge difference density, CDD). 如图2(a) (c)所示, CDD是从复合结构 的电荷中减去 $\mathrm{MoS}_{2}$ 和XS获得. 显然，电荷转移主要发 生在 $\mathrm{MoS}_{2}$ 的 $\mathrm{S}$ 原子层和XS的 $\mathrm{S}$ 原子层之间, 且电子转移 方向是从 $\mathrm{MoS}_{2}$ 到XS, 因此内建电场的方向是从 $\mathrm{MoS}_{2}$ 到 $\mathrm{XS}$. 图2(d)为沿 $z$ 轴方向 3 个复合结构 $x y$ 平面的平均静电 势. 显然, 与XS相比, $\mathrm{MoS}_{2}$ 表现出更低的电势, 因此促 进了电子从 $\mathrm{MoS}_{2}$ 到XS的转移 ${ }^{[33]}$. 有报道指出, 石墨烯 和TMDCs之间的耦合可将电子从电极快速转移到活性 点位，形成的富电子环境可以有效地促进相关反应 ${ }^{[34]}$. 因此，复合结构适宜的带隙和明确的电荷转移将为诸 多应用带来丰富的适用环境.

在对能带进一步调控之前, 先对纯净及 $\mathrm{MoS}_{2}-\mathrm{XS}$ 复合结构的机械特性进行初步探究. 首先, 材料的内部 应变 $\varepsilon$ 可表示为 $\varepsilon=\left(a-a_{0}\right) / a_{0}$, 其中 $a$ 和 $a_{0}$ 分别表示应变和 自由状态下的晶格常数. 图3(a)为复合结构的应变-应 变能变化曲线. 其中, $E_{\mathrm{s}}=\left(E_{\text {strained }}-E_{\text {freestanding }}\right) / n, n$ 表示晶 胞数目. 显然, 所有应变曲线都是准二次曲线, 这意味 

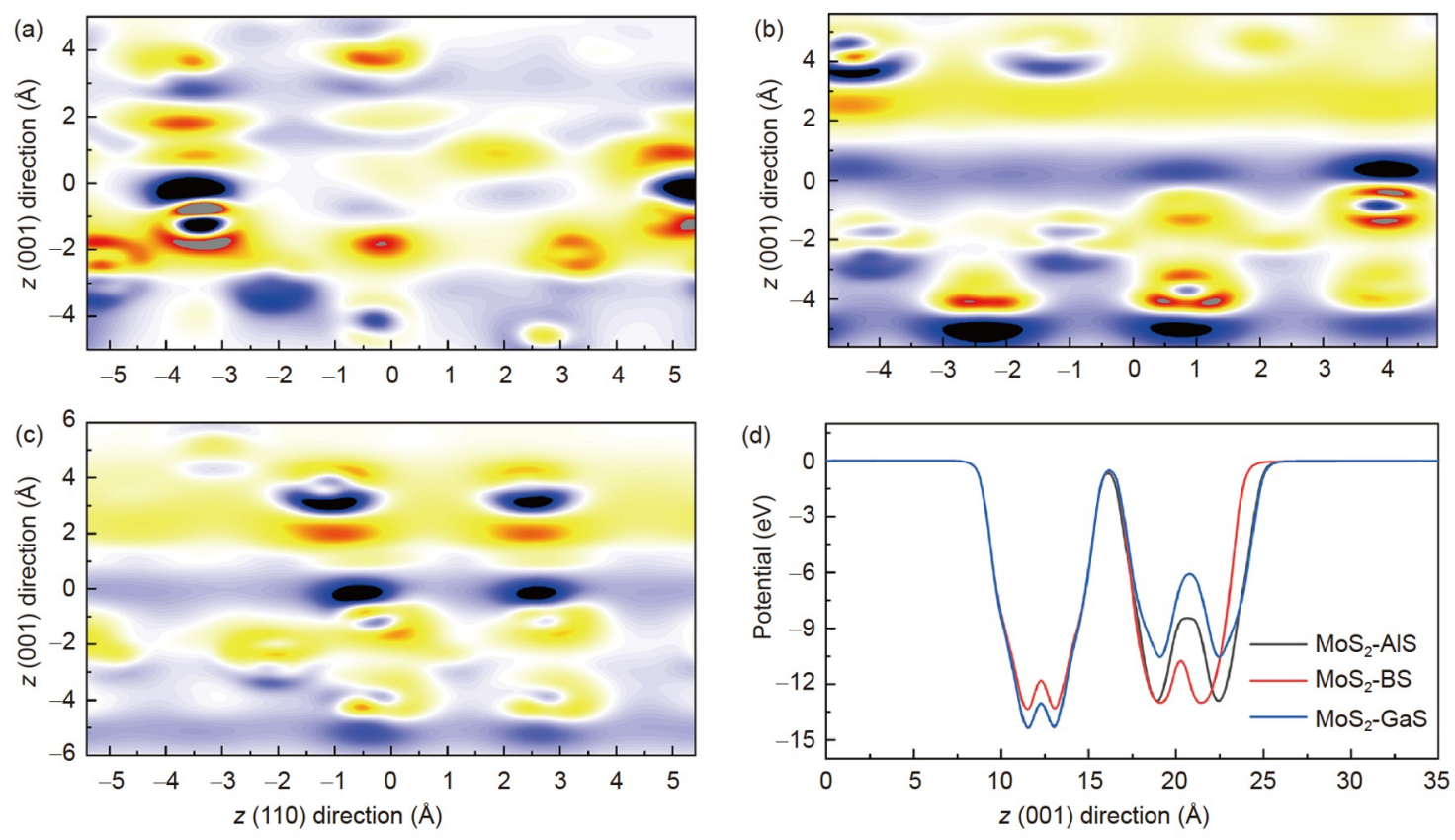

图 2 不同复合结构的电荷密度差分图. (a) $\mathrm{MoS}_{2}-\mathrm{AlS}$; (b) $\mathrm{MoS}_{2}-\mathrm{BS}$; (c) $\mathrm{MoS}_{2}-\mathrm{GaS}$; (d) 沿 $z$ 轴方向3种复合结构的电势曲线图

Figure $2 \mathrm{CDD}$ figures of different heterostructures. (a) $\mathrm{MoS}_{2}-\mathrm{AlS}$; (b) $\mathrm{MoS}_{2}-\mathrm{BS}$; (c) $\mathrm{MoS}_{2}-\mathrm{GaS}$; (d) potential profiles of three heterostructures along $z$ axis

着异质结构在适度的结构形变下都具有一定的延展性 和可逆性. 根据胡克定律, 弹性常数和模量之间的关系 可表示为

$\left[\begin{array}{c}\sigma_{x x} \\ \sigma_{y y} \\ \sigma_{z z}\end{array}\right]=\left[\begin{array}{ccc}C_{11} & C_{12} & 0 \\ C_{12} & C_{22} & 0 \\ 0 & 0 & C_{66}\end{array}\right]\left[\begin{array}{c}\varepsilon_{x x} \\ \varepsilon_{y y} \\ 2 \varepsilon_{x y}\end{array}\right]$.

当施加外部应力时，二维晶体物理性质的变化可 通过特定表达式来描述. $C_{i j}(i, j=1,2,6)$ 表示面内刚度 张量, 其在数值上等于 $E_{\mathrm{s}}$ 的二阶导数(应变能-应变). 使 用标准Voigt表示法, 可写为 $C=\left(1 / S_{0}\right)\left(\partial^{2} E_{\mathrm{s}} /\left(\partial_{\varepsilon i} \partial_{\varepsilon j}\right)\right)^{[35]}$, 其 中 $S_{0}$ 代表结构的平衡面积. 在这种情况下, $C_{i j}$ 可以利用 第一性原理通过以下表达式来计算 ${ }^{[36]}: E_{\mathrm{s}}=\frac{1}{2} C_{11} \varepsilon_{x x}^{2+}$ $\frac{1}{2} C_{2 \varepsilon_{y y}}^{2}+C_{12} \varepsilon_{x x} \varepsilon_{y y}+2 C_{66} \varepsilon_{x y}^{2}$. 沿特定方向施加单轴应变 后可得: $\varepsilon_{y y}=0\left(\varepsilon_{x x}=0\right)$ 和 $E_{\mathrm{s}}=\frac{1}{2} C_{11} \varepsilon_{x x}^{2}\left(\frac{1}{2} C_{11} \varepsilon_{y y}^{2}\right)$. 通过二次 多项式拟合 $E_{\mathrm{s}}$, 并通过相应的系数得到弹性常数 $C_{11} / C_{22}$. 为了合理可信地计算弹性常数, $E_{\mathrm{s}}$ 曲线应变范 围设置为 $-2 \% \leqslant \varepsilon \leqslant 2 \%$, 增量为 $0.5 \%$. 完成上述工作后, 我们首先计算了石墨烯的杨氏模量来初步验证上述计 算流程的可靠性, 并将其与相关文献进行对比. 结果表
明, 石墨烯的杨氏模量为 $344 \mathrm{GPa} n$, 与文献[37,38]报 道的 $340 \mathrm{GPa} \mathrm{nm}$ 吻合, 证明上述采用的参数和流程具 有较高可信度. 纯净 $\mathrm{MoS}_{2} 、 \mathrm{AlS} 、 \mathrm{BS}$ 和 $\mathrm{GaS}$ 的模量分 别计算为 $179.8 、 125.4 、 248.5$ 和120.4 GPa nm. 对于3 种复合结构, $\mathrm{MoS}_{2}-\mathrm{AlS} 、 \mathrm{MoS}_{2}-\mathrm{BS}$ 和 $\mathrm{MoS}_{2}-\mathrm{GaS}$ 的模量 分别为285.4、281.3和297.1 GPa nm，表明异质结构的 模量均大于纯净结构, 因此, $\mathrm{MoS}_{2}$ 与XS复合后相关物 化反应中的稳定性和耐久性会显著增强，并将抑制潜 在的降解或失活现象. 实际上, 这种二维复合结构更优 秀的结构稳定性在诸多实验中已得到证实 ${ }^{[39-41]}$.

由于复合结构中不存在诸如悬空键及不饱和键之 类的强化学键, 两部分之间相互作用相对较弱, 因此材 料的性能受弱 $\mathrm{vdW}$ 力的影响较为明显 ${ }^{[42]}$. 基于此, 首先 分析层间距对光解水性能的影响. 适当的带隙和能带 排列形式是有效利用光能的两个关键因素. 图4(a), (b) 为 $\mathrm{MoS}_{2}-\mathrm{AlS}$ 能带边缘及带隙的变化情况，以层间距平 衡位置为零点, 变化范围为-2.5 1.0 ̊. 图4(b)显示, 复 合材料的带隙会随着层间距的减小而显著降低，其 $\mathrm{VBM}$ 上移且CBM下移. 图4(a)中, 将还原电势 $\mathrm{H}^{+} / \mathrm{H}_{2}$ 用 上直线标出, 氧化电势 $\mathrm{O}_{2} / \mathrm{H}_{2} \mathrm{O}$ 用下直线标出, 其间距为 $1.23 \mathrm{eV}$. 一般而言, 能自发发生氧化还原反应的半导体 带隙应 $>1.23 \mathrm{eV}$, 且 $V B M$ 和 $C B M$ 分别位于两条直线外 


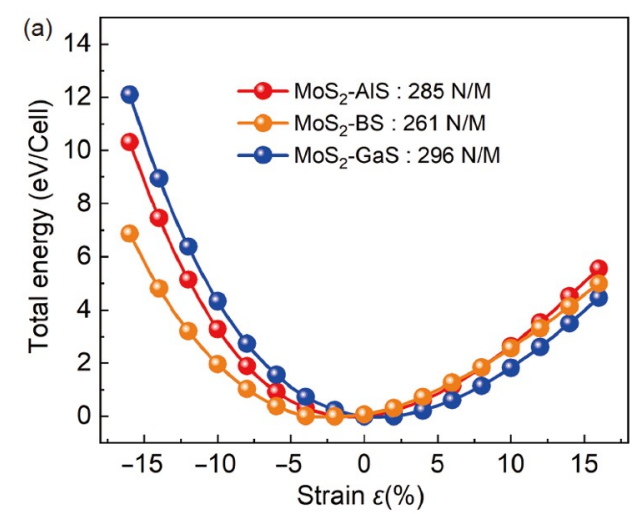

(c)

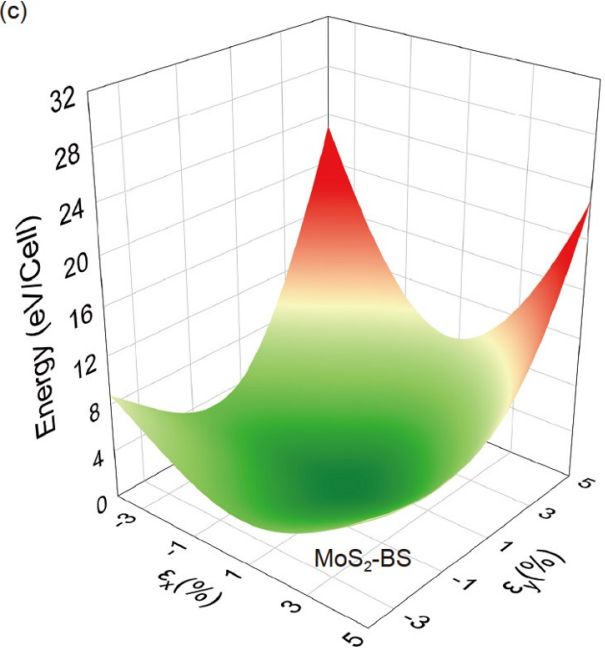

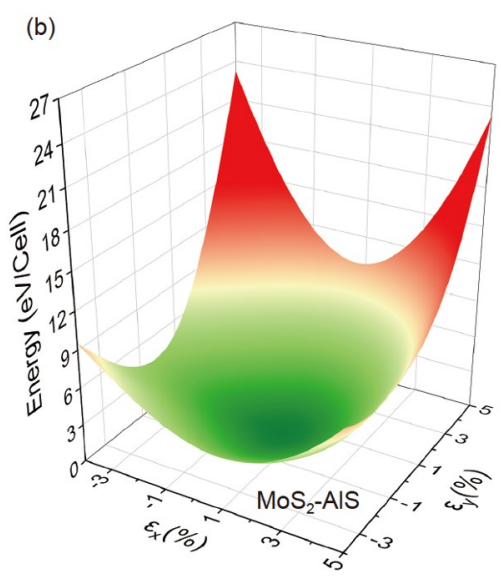

(d)

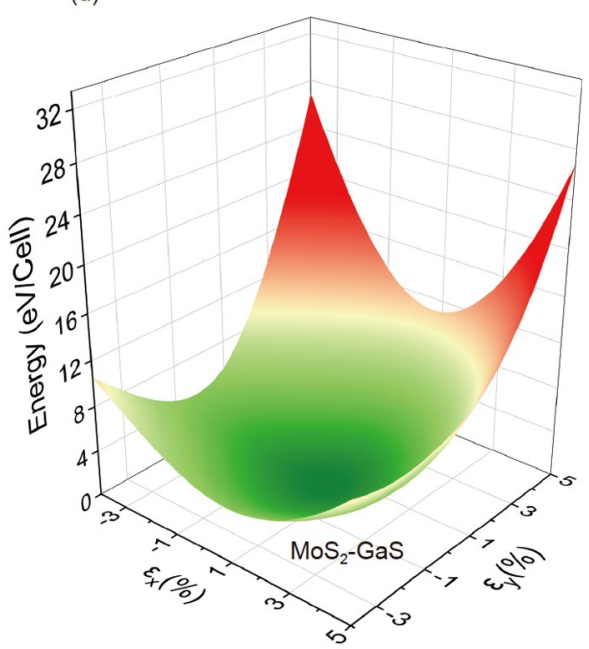

图 3 复合结构对不同应变的响应. (a) $\mathrm{MoS}_{2}-\mathrm{XS}$ 复合结构的应变-应变能曲线; $\mathrm{MoS}_{2}-\mathrm{AlS}(\mathrm{b}) 、 \mathrm{MoS}_{2}-\mathrm{BS}(\mathrm{c})$ 和MoS $\mathrm{M}_{2}-\mathrm{GaS}(\mathrm{d})$ 的 $3 \mathrm{D}$ 应变-应变能曲面 Figure 3 Responses of heterostructures to different strains. (a) Strain-strain energy curves of $\mathrm{MoS}_{2}$-XS composite structures; 3D strain-strain energy surfaces of $\mathrm{MoS}_{2}$-AlS (b), $\mathrm{MoS}_{2}$-BS (c) and $\mathrm{MoS}_{2}-\mathrm{GaS}(\mathrm{d})$

侧. 最理想的能带排列形式应是II型, 在图4(a)中将其用 浅背底标出. 此时, 对于 $\mathrm{MoS}_{2}-\mathrm{AlS}$ 复合材料, 最适于光 解水的层间距应在 $-1 \sim 1 \AA$ 之间变化. 这是因为, 在这个 区间内的 $\mathrm{MoS}_{2}-\mathrm{A} 1 \mathrm{~S}$ 不仅有II型能带排列形式，而且 $\mathrm{VBM}$ 和CBM分别位于 $\mathrm{O}_{2} / \mathrm{H}_{2} \mathrm{O}$ 和 $\mathrm{H}^{+} / \mathrm{H}_{2}$ 两侧, 是光解水 反应的最佳选择. 而对于图4(c), (d)的 $\mathrm{MoS}_{2}-\mathrm{BS}$, 情况 发生了变化, 不论层间距如何变化, 其VBM总是位于氧 化电位和还原电位之间，即其氧化能力不足以推动自 发的光解水反应. 对于图4(e), (f)的 $\mathrm{MoS}_{2}-\mathrm{GaS}$, 其能够 发生自发光解水反应的间距范围应为 $-1.58 \sim 1.22 \AA$, 显 著小于 $\mathrm{MoS}_{2}$-AlS结构. 3 种复合结构在 $d_{0} \pm 1.6 \AA$ 层间距 下的能带结构在图S1中给出. 基于此, 当层间距变化时, $\mathrm{MoS}_{2}$-AlS结构有着最宽泛的II型能带区间, 最适于光解 水催化剂的制备.

如上所述，适当的带隙和能带排布形式是高效利
用光能的两个关键因素. 文献[43]指出，通过外部电场 进行能带调节是达成该目标的另一种方式. 当半导体 的能带边缘受到电场调控时, $\mathrm{VBM} / \mathrm{CBM}$ 的变化将不 可避免, 活性材料的氧化还原能力也会受到影响. 图5 显示了不同电场下 3 种异质结带边变化情况，以达到调 节CBM/VBM排列并最大化利用光能的目的. 图5(a)显 示, 在外加电场的情况下, 复合材料中 $\mathrm{MoS}_{2} / \mathrm{AlS}$ 的带隙 变化很小, 但 $\mathrm{CBM} / \mathrm{VBM}$ 的相对位置受到显著影响. 当 施加正向电场时，AlS的边缘位置会向下移动．当外部 电场达到 -0.25 和 $0.25 \mathrm{~V} / \AA$ 时，带隙将减小至 $0 \mathrm{eV}$. 在 $-0.05 \mathrm{~V} / \AA$ 时，带隙将达到最大值 $1.76 \mathrm{eV}$. 需要注意的 是, 电场在 $-0.03 \sim 0.02 \mathrm{~V} / \AA$ 时, $\mathrm{MoS}_{2}-\mathrm{AlS}$ 复合结构有着 II型异质结的能带排列形式, 并且其带边位于水解氧化 还原电位的外侧，这是最理想的情况. 在这个范围内, $\mathrm{MoS}_{2}$ 的带边高于 $\mathrm{AlS}$, 因此 $\mathrm{MoS}_{2}(\mathrm{AlS})$ 的VBM(CBM)分 

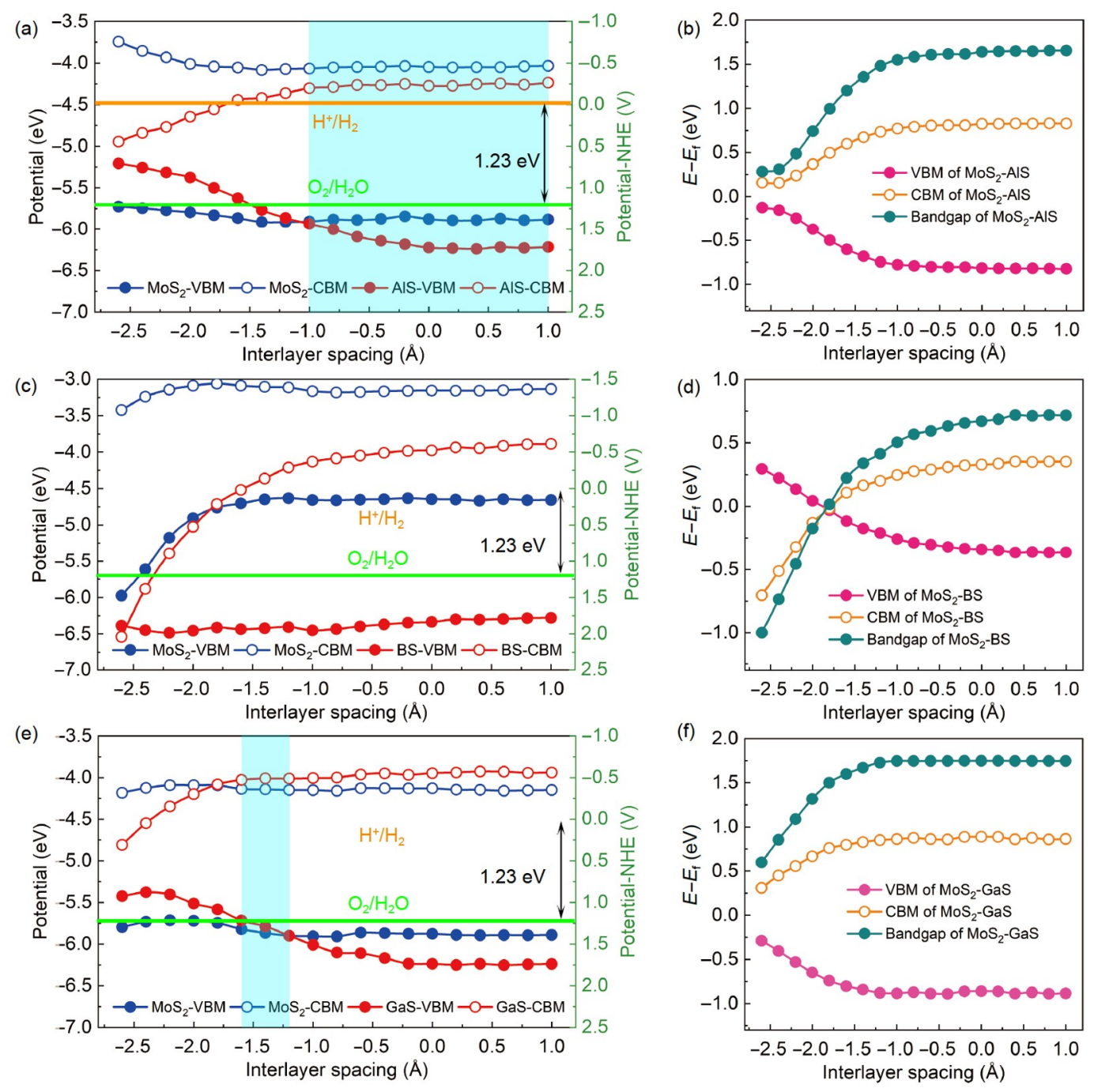

图 4 复合结构的层间距对能带排列及带隙的影响. (a, b) $\mathrm{MoS}_{2}-\mathrm{AlS} ;(\mathrm{c}, \mathrm{d}) \mathrm{MoS}_{2}-\mathrm{BS} ;(\mathrm{e}, \mathrm{f}) \mathrm{MoS}_{2}-\mathrm{GaS}$. 横坐标取值为实际层间距减去结合能最 小时的层间距

Figure 4 The effect of the interlayer spacing of the heterostructure on the band alignment and band gap. (a, b) $\operatorname{MoS}_{2}-\mathrm{AlS}_{\text {; }}(\mathrm{c}, \mathrm{d}) \mathrm{MoS}_{2}-\mathrm{BS}$; (e, f) MoS ${ }_{2}$ $\mathrm{GaS}$. The abscissa is taken as the actual interlayer spacing minus the interlayer spacing when the binding energy is minimum

别成为 $\mathrm{MoS}_{2}-\mathrm{AlS}$ 的 $\mathrm{VBM}(\mathrm{CBM})$. 对于图 5(c)，(e)的 $\mathrm{MoS}_{2}-\mathrm{BS} / \mathrm{GaS}$, 在整个电场变化范围内均不能在具备 II 型异质结的同时满足自发光解水的氧化还原电位. 3 种 复合结构在 $\pm 0.1 \mathrm{~V} / \AA \AA$ 电场下的能带结构在图 $\mathrm{S} 2$ 中给出. 需要说明的是, 虽然I型异质结不适于光解水催化剂, 但 其载流子的快速复合可以应用于某些特殊的光电器件, 同样有着广阔的应用潜力 ${ }^{[44,45]}$. 我们注意到, 这种能带 变化形式与随后应变引起的变化有很大的不同.

图6为对 $\mathrm{MoS}_{2}-\mathrm{XS}$ 复合结构施加单轴应变后 $\mathrm{CBM} /$ $\mathrm{VBM}$ 的变化趋势，为进一步调控异质结的能带排列和 最大化利用光能提供了有意义的参考. 从图6(a), (b)可
以看出, $\mathrm{MoS}_{2}-\mathrm{AlS}$ 的带隙在施加应变后减小, 表明带隙 和带边都会随外部应力的变化而变化. 在此, 我们同样 关注能够使 $\mathrm{MoS}_{2}$-AlS复合结构自发进行光解水反应的 区域. 如图6(a)所示, 当对 $\mathrm{MoS}_{2}$-AlS复合结构在浅色区 域( $-6 \%$ 2\%)内施加应变时, 其带隙会随着外部应变的 增加而显著减小. 复合结构的 $\operatorname{VBM}(\mathrm{CBM})$ 是 $\mathrm{MoS}_{2}(\mathrm{AlS})$ 的 $\operatorname{VBM}(\mathrm{CBM})$, 因此在整个应变范围内均表现出II型能 带排列形式. 对于图6(c) (f)中所示的 $\mathrm{MoS}_{2}-\mathrm{BS} / \mathrm{GaS}$ 复 合结构，虽然带隙与 $\mathrm{MoS}_{2}$-AlS有类似的变化趋势，也 同样随应力的增加而减小, 但在整个加压范围内, 异质 结均不能在具备II型异质结的同时满足自发进行光解 

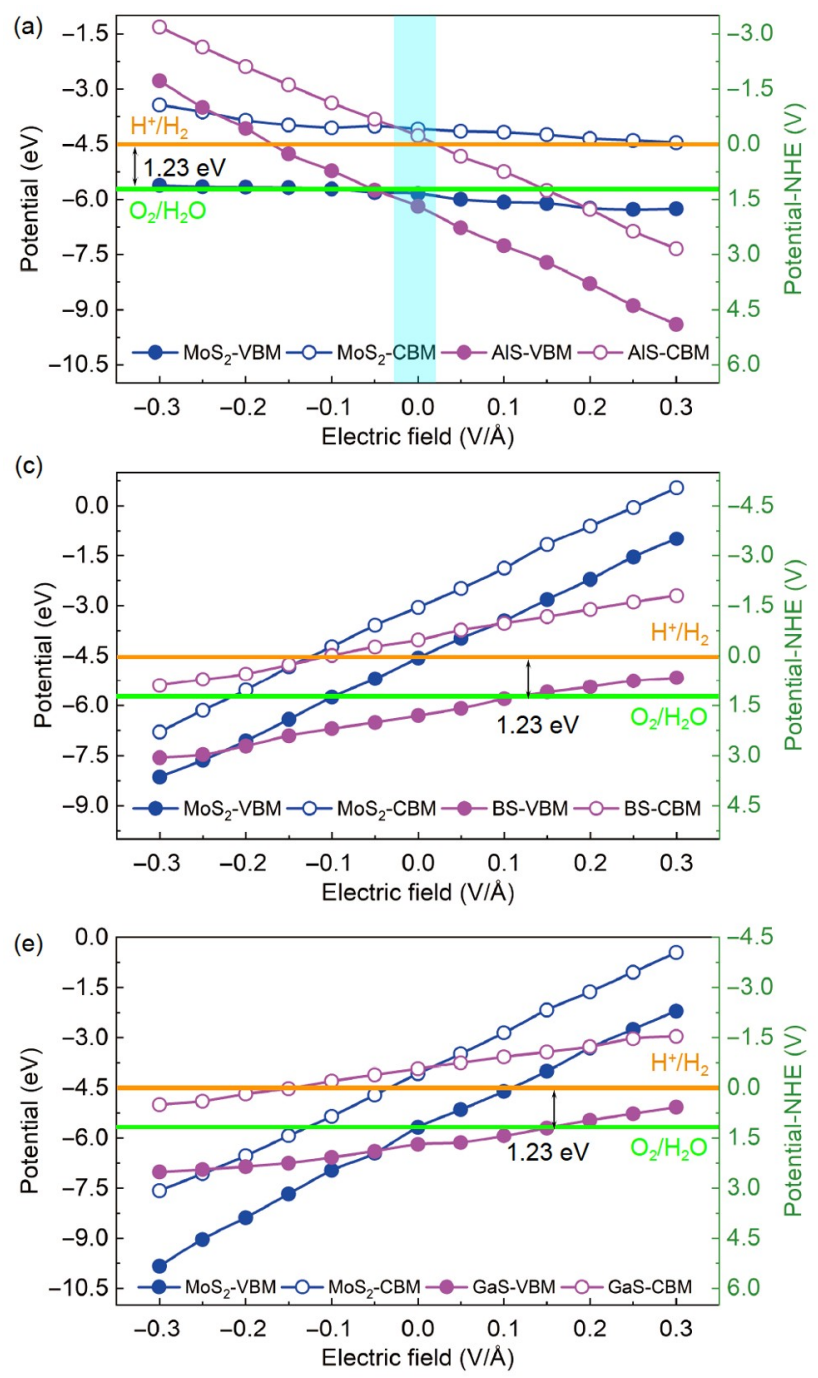

(b)

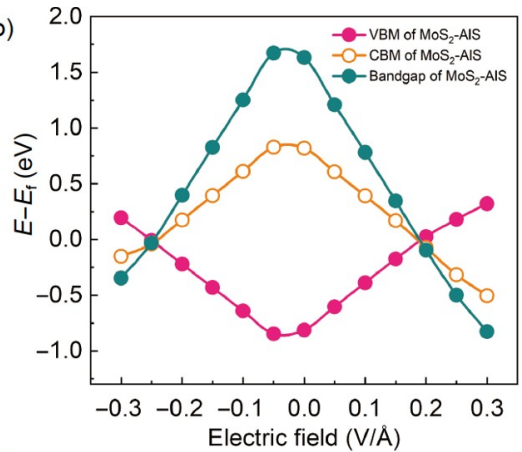

(d)
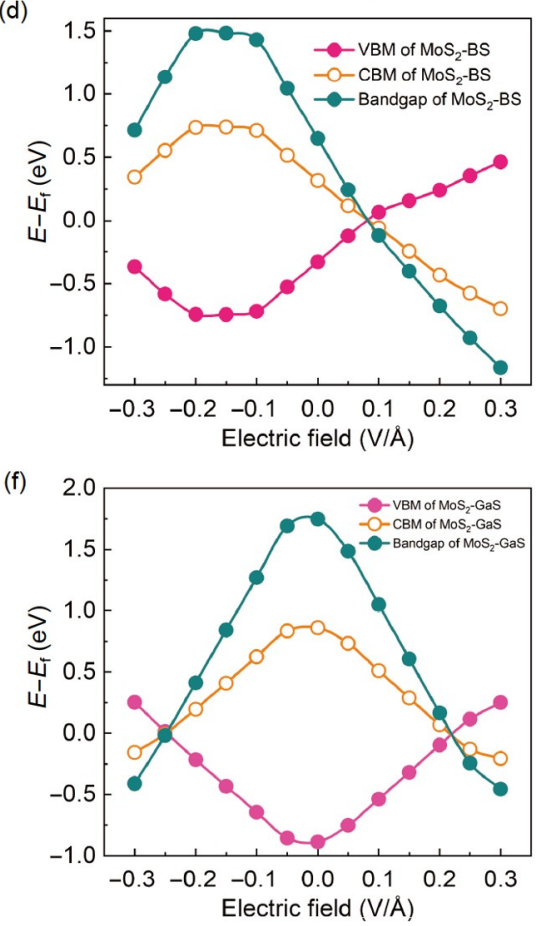

图 $5-0.3 \sim 0.3 \mathrm{~V} / \AA$ 的电场对复合结构能带排列的影响. (a, b) $\mathrm{MoS}_{2}-\mathrm{AlS}$; (c, d) $\mathrm{MoS}_{2}-\mathrm{BS}$; (e, f) $\mathrm{MoS}_{2}-\mathrm{GaS}$

Figure 5 The influence of the electric field from -0.3 to $0.3 \mathrm{~V} / \AA$ on band edges alignment of heterostructures. (a, b) $\mathrm{MoS}_{2}-\mathrm{AlS}$; (c, d) MoS $2-\mathrm{BS}$; (e, f) $\mathrm{MoS}_{2}-\mathrm{GaS}$

水的氧化还原电位. 3 种复合结构在 $\pm 4 \%$ 应变下的能带 结构在图S3中给出. 总之, $\mathrm{MoS}_{2}-\mathrm{XS}$ 的电子性质会受外 部应变的显著影响. 我们对应变如何影响带隙和带边 进行了详细的分析和讨论. 从变间距、加电场到加应 变, $\mathrm{MoS}_{2}$-AlS体现了良好的催化活性, 都存在具备II型 异质结的同时满足理想氧化还原电位的区域. 因此, 就 光解水而言, 我们认为 $\mathrm{MoS}_{2}$-AlS 是最理想的选择.

除以上讨论的能带结构外，光解水过程中量子效 率与载流子的分离转移同样密切联系, 而载流子迁移 率又与有效质量直接相关 ${ }^{[46]}$. 理论上，可以通过拟合 VBM或CBM的抛物线获得有效空穴质量 $\left(m_{\mathrm{h}}{ }^{*}\right)$ 或有效 电子质量 $\left(m_{\mathrm{e}}{ }^{*}\right)$. 根据半导体理论, 可通过下式获得半导
体的 $m_{\mathrm{h}}{ }^{*}$ 或 $m_{\mathrm{e}}{ }^{*[47]}: \frac{1}{m_{i}^{*}}=\frac{1}{\hbar^{2}}\left(\frac{\partial^{2} E}{\partial k_{i}^{2}}\right) \hbar$, 其中 $E$ 是能量, $k$ 是 波矢. 为方便比较, 能带计算方向统一确定为 $\mathrm{G} \rightarrow \mathrm{K}$ 和 $\mathrm{G} \rightarrow \mathrm{M}$. 表 1 总结了沿两个方向 $\mathrm{MoS}_{2}$ 和 $X \mathrm{~S}$ 的 $m_{\mathrm{h}}{ }^{*}$ 和 $m_{\mathrm{e}}{ }^{*}$. 一般而言，载流子密度和迁移率是光电材料相关 应用中的两个重要参数, 且与有效质量高度相关. 其 中, 载流子密度与有效质量成正比, 可以表示为 $n=\frac{g_{v} m^{*} k_{\mathrm{B}} T}{\pi \hbar^{2}} \ln \left(\exp \left(-\frac{e \varphi}{k_{\mathrm{B}} T}\right)+1\right), k_{\mathrm{B}} 、 T 、 g_{v}$ 和 $\varphi$ 分别是玻 尔兹曼常数、温度、简并度和能量差. 然而, 迁移率与 有效质量却成反比, 可以写为 $\mu=\frac{e \tau}{m^{*}} \hbar$, 其中 $e 、 m^{*}$ 和 $\tau$ 分 

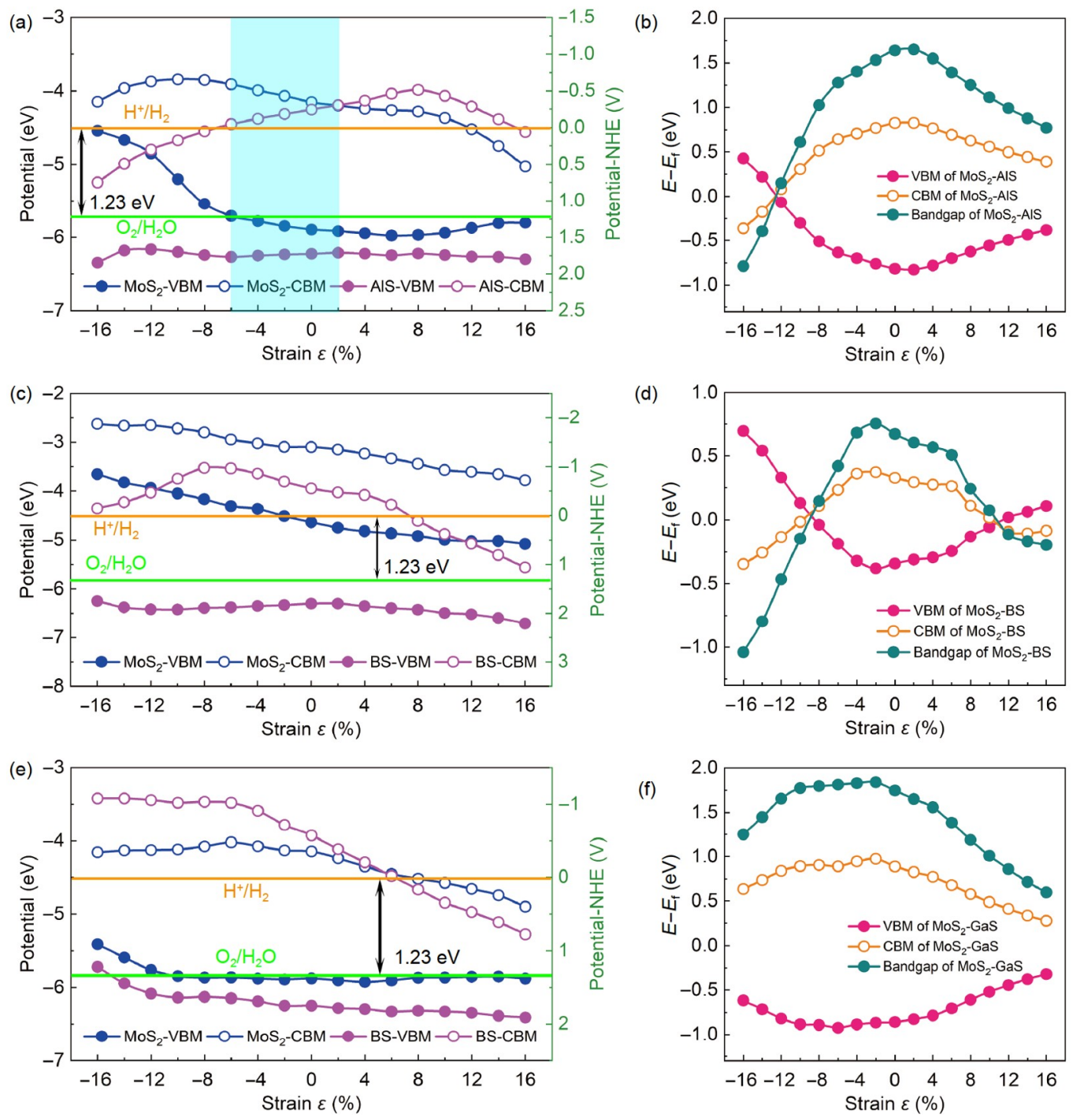

图 $6-16 \%$ 16\%的外部应变对复合结构能带排列的影响. (a, b) $\mathrm{MoS}_{2}-\mathrm{AlS}$; (c, d) $\mathrm{MoS}_{2}-\mathrm{BS}$; (e, f) $\mathrm{MoS}_{2}-\mathrm{GaS}$

Figure 6 The influence of the external strain from $-16 \%$ to $16 \%$ on band edges alignment of heterostructures. (a, b) $\mathrm{MoS}_{2}-\mathrm{AlS} ;(\mathrm{c}, \mathrm{d}) \mathrm{MoS}_{2}-\mathrm{BS}$; (e, f) $\mathrm{MoS}_{2}-\mathrm{GaS}$

别是单位电荷、有效质量和弛豫时间 ${ }^{[48]}$. 因此, 当有效 质量为唯一变量时, $n$ 和 $\mu$ 的变化趋势恰好相反. 从表 1 可以看出，纯净 $\mathrm{MoS}_{2}$ 沿 $\mathrm{G} \rightarrow \mathrm{K}$ 方向的 $m_{\mathrm{h}}{ }^{*}$ 和 $m_{\mathrm{e}}{ }^{*}$ 大于所有 $\mathrm{XS}$, 而 $\mathrm{GaS}$ 在两个方向上都有最小的有效电子质量, 说 明其载流子可以更快地迁移到材料表面并参与相关的 氧化还原反应. 一般而言, 半导体在带边的离散特性影 响载流子的有效质量, 相对平坦的能带边缘意味着较 高的有效质量. 图1表明, GaS沿着两个方向离散的能 带结构将带来更小的有效质量. 虽然AlS或GaS沿着两 个方向上的 $m_{\mathrm{h}}{ }^{*}$ 和 $m_{\mathrm{e}}{ }^{*}$ 都在不同程度上小于 $\mathrm{MoS}_{2}$, 并且 根据图1可知，4种纯净材料都满足自发氧化还原反应
的电位, 然而其 $\mathrm{VBM}$ 和 $\mathrm{CBM}$ 都来源于 $\mathrm{S}$ 原子, 这将带来 显著的载流子复合, 不利于相关反应的顺利进行. 这正 是将 $\mathrm{MoS}_{2}$ 和XS进行复合处理的主要目的: 抑制载流子 复合. 接下来, 将进一步说明复合结构如何影响相关结 构的有效质量.

图7为 $\mathrm{MoS}_{2}-\mathrm{XS}$ 异质结在不同应变下有效质量的 变化趋势. 总体而言, 复合结构中的有效质量在不同程 度上均低于纯净结构. 举例说明, $\mathrm{MoS}_{2}$ 沿 $\mathrm{G} \rightarrow \mathrm{K}$ 方向的 有效空穴质量均高于 $5 m_{0}$, 而在 3 种复合结构中, 不管应 变如何变化，总体的空穴有效质量均未超过 $2 m_{0}$. 根据 图6, 这里主要分析-6\% 2\%的浅色区域. 在图7(a)中, 
表 1 沿 $\mathbf{G} \rightarrow \mathbf{K}$ 和 $\mathbf{G} \rightarrow \mathbf{M}$ 方向单层结构的电子/空穴有效质量

Table 1 Effective masses of electrons and holes along $\mathrm{G} \rightarrow \mathrm{K}$ and $\mathrm{G} \rightarrow \mathrm{M}$ for monolayer structures

\begin{tabular}{ccccc}
\hline & $\mathrm{MoS}_{2}$ & AlS & BS & GaS \\
\hline $\mathrm{VBM}(\mathrm{G} \rightarrow \mathrm{K}) / m_{\mathrm{h}}{ }^{*}$ & 5.118 & 0.893 & 3.143 & 0.851 \\
$\mathrm{VBM}(\mathrm{G} \rightarrow \mathrm{M}) / m_{\mathrm{h}}{ }^{*}$ & -0.927 & 0.910 & 3.348 & 0.845 \\
$\mathrm{CBM}(\mathrm{G} \rightarrow \mathrm{K}) / m_{\mathrm{e}}{ }^{*}$ & -6.936 & 0.725 & 0.638 & 0.216 \\
$\mathrm{CBM}(\mathrm{G} \rightarrow \mathrm{M}) / m_{\mathrm{e}}{ }^{*}$ & 0.691 & 0.922 & 4.663 & 0.215 \\
\hline
\end{tabular}

首先要注意的是相对较低的 $m_{\mathrm{e}}{ }^{*}$ 值, 在整个浅色范围内, $m_{\mathrm{e}}{ }^{*}$ 可以在 $-6 \%$ 的应变下达到 $-0.15 m_{0}$ 的极低值并远小 于纯净结构. 这意味着复合结构的空穴迁移率将远高 于纯净结构, 从而有着更优秀的氧化活性. 这是一个值 得注意的现象, 虽然VBM完全由 $\mathrm{MoS}_{2}$ 贡献(图1(a)), 然 而 $\mathrm{MoS}_{2}-\mathrm{AlS}$ 复合结构却比纯净 $\mathrm{MoS}_{2}$ 有着更低的有效 质量和更好的输运特性. 这主要是由于弱 $\mathrm{vdW}$ 力影响 了相应的能带结构, 从而利于载流子的高效输运. 当对 $\mathrm{MoS}_{2}-\mathrm{AlS}$ 复合结构施加正向应变时，虽然有效质量同 样有着显著的降低, 但幅度小于负向应变. 因此, 我们 认为, 对 $\mathrm{MoS}_{2}$ - $\mathrm{AlS}$ 施加压应变将显著提高材料的光解 水活性, 因为这种情况下复合材料不仅能保持II型异质 结特性、满足自发光解水电位，而且能够显著降低电 子/空穴有效质量, 从而提高反应的整体催化活性.

根据以上讨论发现，复合材料是相当优秀的光解 水催化剂, 尤其是施加压应变后, 其性能将得到进一步 提升. 此外, 异质结构在太阳能电池方面同样有着广阔 的应用潜力, 并被认为是价廉高效的光伏材料 ${ }^{[49]}$. 因此, 有必要对基于 $\mathrm{MoS}_{2}$-XS异质结的太阳能电池进行初探 性研究. 根据定义, 太阳能电池材料的性能可以通过能 量转换效率(power conversion efficiency, PCE)来定量评 估 ${ }^{[50]}$ :

$\eta=\frac{0.65\left(E_{\mathrm{g}}-\Delta E_{\mathrm{c}}-0.3\right) \int_{E_{\mathrm{g}}}^{\infty} \frac{P(\hbar \omega)}{\hbar \omega} \mathrm{d}(\hbar \omega)}{\int_{0}^{\infty} P(\hbar \omega) \mathrm{d}(\hbar \omega)} \hbar$,

其中, 常数 0.65 是填充因子, $P(\hbar \omega)$ 表示AM1.5下太阳能 通量 $\left(\mathrm{W} \mathrm{m}^{-2} \mathrm{eV}^{-1}\right)$ 与光子能量 $(\hbar \omega)$ 之间的关系 ${ }^{[51]}, E_{\mathrm{g}}$ 和 $\Delta E_{\mathrm{c}}$ 分别是施主带隙和导带偏移(conduction band offset, $\mathrm{CBO}$ ). 由此可知, 异质结的能带排列可直接确定PCE. 图8是在上述条件下计算出的PCE等高图, 其 $x$ 和 $y$ 轴分 别是施主带隙和 $\mathrm{CBO}$ ，每条轮廓线代表一个恒定的 $\mathrm{PCE}$ 值, 以下讨论主要集中在 $\mathrm{CBO}$ 为 $0 \sim 1 \mathrm{eV}$ 范围内. 考
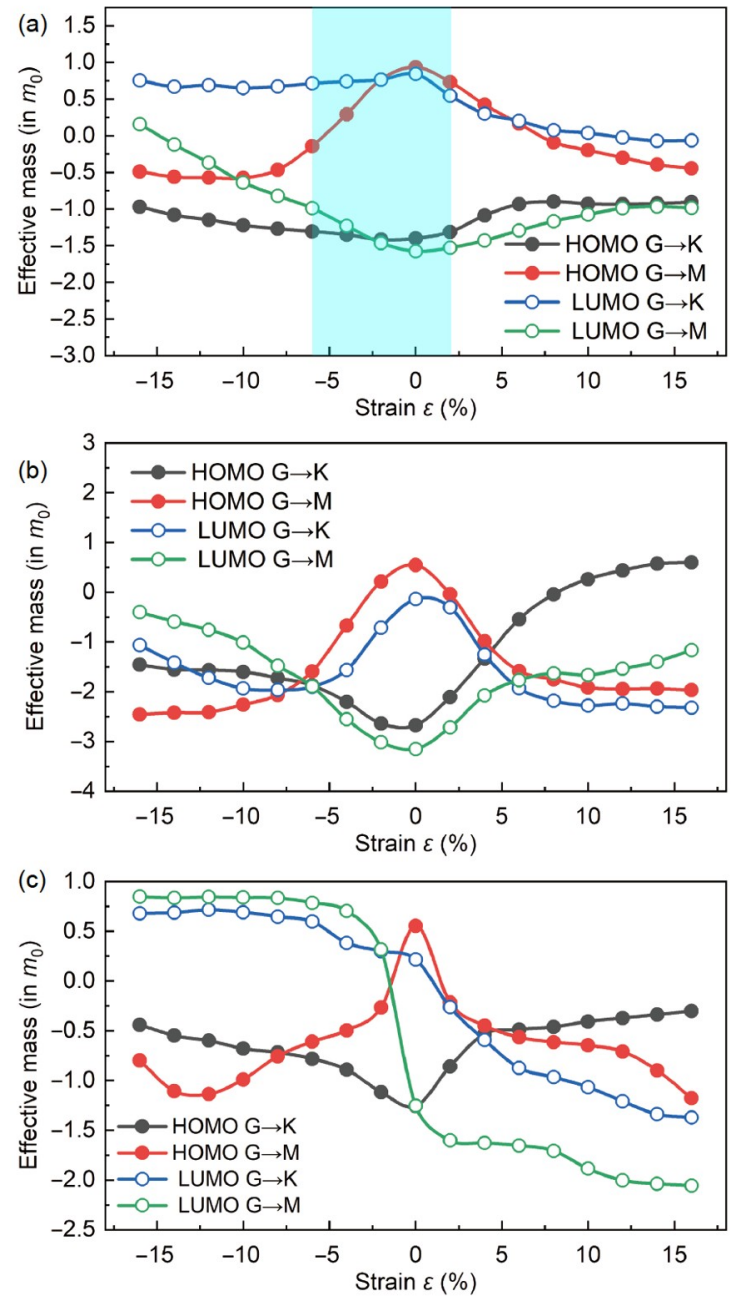

图 73 个复合结构在 $-0.16 \sim 0.16$ 应变范围内有效电子质量和空穴质 量的变化趋势. (a) $\mathrm{MoS}_{2}-\mathrm{AlS}$; (b) $\mathrm{MoS}_{2}-\mathrm{BS}$; (c) $\mathrm{MoS}_{2}-\mathrm{GaS}$. $m_{0}$ 为自由 电子质量

Figure 7 Effective electron/hole mass evolution of the three heterostructures in the strain range from -0.16 to 0.16 . (a) $\mathrm{MoS}_{2}$-AlS; (b) $\mathrm{MoS}_{2}-\mathrm{BS}$; (c) $\mathrm{MoS}_{2}-\mathrm{GaS} . m_{0}$ is the mass of a free electron

虑到自由状态下的复合结构已经是II型异质结, 这里关 注自由状态下的复合结构. 首先，对于 $\mathrm{MoS}_{2}-\mathrm{AlS}$ ，其 PCE约为 $17.1 \%$, 而 $\mathrm{MoS}_{2}-\mathrm{GaS}$ 则进一步增加到 $18.2 \%$. 对于 $\mathrm{MoS}_{2}-\mathrm{BS}$, 其PCE高达 $20.4 \%$. 虽然 $\mathrm{MoS}_{2}-\mathrm{BS}$ 在上文 一系列计算分析中均被证实不适于光解水催化剂的应 用, 但在太阳能电池应用方面却展现了出色的效率. 值 得注意的是，该值非常接近甚至优于已报道的异质结 构太阳能电池，如PCBM/CBN $(10 \% \sim 20 \%)^{[52] 、 g-S i C / ~}$ $\mathrm{GaN}(12 \% \sim 20 \%)^{[53]} 、 \mathrm{SnO}_{2} / \mathrm{TiO}$ 梯度异质结 $(18.08 \%)^{[54]}$ 、 $2 \mathrm{D} \mathrm{GaX} / \mathrm{SnS}_{2}(16 \%)^{[55]}$ 、钙钛矿基异质结构 $(21.02 \%)^{[56]}$ 


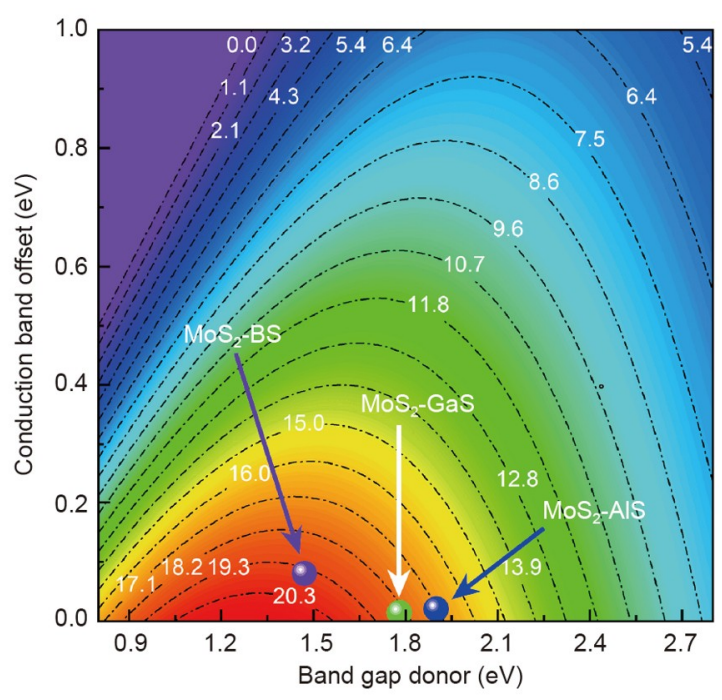

图 $8 \mathrm{MoS}_{2}$-XS在自由状态下的PCE取值. 考虑到实际情况, 效率限 制为 $21 \%$

Figure 8 PCE value of $\mathrm{MoS}_{2}$-XS in freestanding. Considering the actual situation, the efficiency is limited to $21 \%$

和BP $/ \mathrm{SnSe}(17.24 \%)^{[57]}$. 换言之, 当 $\mathrm{MoS}_{2}-\mathrm{BS}$ 应用于太阳 能电池材料时，其不仅能保持II型异质结特性，而且可
输出相对较高的PCE.

\section{2 结论}

近年来，基于TMDCs复合结构的研究显示了广阔 的前景. 当结合两种材料独特的物化属性后, 有望满足 人们对某项性能的特殊要求. 本研究提出了一种具有 本征II型能带排列的 $\mathrm{MoS}_{2}-\mathrm{XS}$ 异质结构, 并通过第一性 原理系统地研究了 3 类异质结构的电子结构、能带排 列、电荷分布，以及其对层间距变化、外加电场、外 加应变的响应. 3种复合材料的能带结构是灵活可调 的, 有利于光催化材料或光电器件的应用. II型能带结 构的排列形式可促进界面载流子的分离转移, 并抑制 电子-空穴对的复合, 从而实现更好的光能利用. 还通 过一系列外部作用研究了 3 种异质结构的电子结构和 相关性质, 为后续进一步的实验研究提供了有价值的 参考. 其中, $\mathrm{MoS}_{2}-\mathrm{AlS}$ 是优秀的光催化剂, 尤其是施加 压应变后, 其光解水性能将得到进一步提升. 此外, $\mathrm{MoS}_{2}$-BS 异质结的PCE可高达 $20.4 \%$ ，有望应用于太 阳能电池. 总之, 我们认为, 这项工作为二维复合材料 如何高效利用光能的方式和途径提供了有意义的参考.

\section{参考文献}

1 Yang S, Zhang P, Nia A S, et al. Emerging 2D materials produced via electrochemistry. Adv Mater, 2020, 32: 1907857

2 Li Z, Lü Y, Ren L, et al. Efficient strain modulation of 2D materials via polymer encapsulation. Nat Commun, 2020, 11: 1151

3 Kanahashi K, Pu J, Takenobu T. 2D materials for large-area flexible thermoelectric devices. Adv Energy Mater, 2020, 10: 1902842

4 Wang J, Luo Y, Cai X, et al. Multiple regulation over growth direction, band structure, and dimension of monolayer $\mathrm{WS}_{2}$ by a quartz substrate. Chem Mater, 2020, 32: 2508-2517

5 Li P, Zhang Z. Self-powered 2D material-based $\mathrm{pH}$ sensor and photodetector driven by monolayer $\mathrm{MoSe}_{2}$ piezoelectric nanogenerator. ACS Appl Mater Interfaces, 2020, 12: 58132-58139

6 Cheng Y, Li H, Liu B, et al. Vertical 0D-perovskite/2D-MoS ${ }_{2}$ van der Waals heterojunction phototransistor for emulating photoelectricsynergistically classical pavlovian conditioning and neural coding dynamics. Small, 2020, 16: 2005217

7 Lee D, Hwang E, Lee Y, et al. Multibit $\mathrm{MoS}_{2}$ photoelectronic memory with ultrahigh sensitivity. Adv Mater, 2016, 28: 9196-9202

8 Kumar R, Das D, Singh A K. $\mathrm{C}_{2} \mathrm{~N} / \mathrm{WS}_{2}$ van der Waals type-II heterostructure as a promising water splitting photocatalyst. J Catal, 2018, 359: 143150

9 Rosman N N, Yunus R M, Minggu L J, et al. Photocatalytic properties of two-dimensional graphene and layered transition-metal dichalcogenides based photocatalyst for photoelectrochemical hydrogen generation: An overview. Int J Hydrog Energy, 2018, 43: 18925-18945

10 Jana M, Xu R, Cheng X B, et al. Rational design of two-dimensional nanomaterials for lithium-sulfur batteries. Energy Environ Sci, 2020, 13: 1049-1075

11 David A, Rakyta P, Kormányos A, et al. Induced spin-orbit coupling in twisted grapheme-transition metal dichalcogenide heterobilayers: Twistronics meets spintronics. Phys Rev B, 2019, 100: 085412

12 Han W. Perspectives for spintronics in 2D materials. APL Mater, 2016, 4: 032401

13 Liu Y, Duan X, Huang Y, et al. Two-dimensional transistors beyond graphene and TMDCs. Chem Soc Rev, 2018, 47: 6388-6409

14 Greben K, Arora S, Harats M G, et al. Intrinsic and extrinsic defect-related excitons in TMDCs. Nano Lett, 2020, 20: 2544-2550

15 Wu L, Guo J, Wang Q, et al. Sensitivity enhancement by using few-layer black phosphorus-graphene/TMDCs heterostructure in surface plasmon resonance biochemical sensor. Sens Actuat B-Chem, 2017, 249: 542-548 
16 Yu Z, Ong Z Y, Li S, et al. Analyzing the carrier mobility in transition-metal dichalcogenide $\mathrm{MoS}_{2}$ field-effect transistors. Adv Funct Mater, 2017, 27: 1604093

17 Zhang K, Zhang T, Cheng G, et al. Interlayer transition and infrared photodetection in atomically thin type-II $\mathrm{MoTe}_{2} / \mathrm{MoS}_{2}$ van der Waals heterostructures. ACS Nano, 2016, 10: 3852-3858

18 Kang J, Sahin H, Peeters F M. Tuning carrier confinement in the $\mathrm{MoS}_{2} / \mathrm{WS}_{2}$ lateral heterostructure. J Phys Chem C, 2015, 119: $9580-9586$

19 Obaidulla S M, Habib M R, Khan Y, et al. $\mathrm{MoS}_{2}$ and perylene derivative based type-II heterostructure: Bandgap engineering and giant photoluminescence enhancement. Adv Mater Interfaces, 2020, 7: 1901197

20 Zhou H, Cai W, Li J, et al. $\mathrm{C}_{2} \mathrm{~N} /$ BlueP van der Waals hetero-structure: An efficient photocatalytic water splitting 2D material. Phys Chem Chem Phys, 2020, 22: 1485-1492

21 Vu T V, Hieu N V, Phuc H V, et al. Graphene/WSeTe van der Waals heterostructure: Controllable electronic properties and Schottky barrier via interlayer coupling and electric field. Appl Surf Sci, 2020, 507: 145036

22 Liu B, Liao Q, Zhang X, et al. Strain-engineered van der Waals interfaces of mixed-dimensional heterostructure arrays. ACS Nano, 2019, 13: 9057-9066

23 Demirci S, Avazlı N, Durgun E, et al. Structural and electronic properties of monolayer group III monochalcogenides. Phys Rev B, 2017, 95: 115409

24 Pan J, Shao X, Xu X, et al. Organic dye molecules sensitization-enhanced photocatalytic water-splitting activity of $\mathrm{MoS}_{2}$ from first-principles calculations. J Phys Chem C, 2020, 124: 6580-6587

25 Liao J, Sa B, Zhou J, et al. Design of high-efficiency visible-light photocatalysts for water splitting: $\mathrm{MoS}_{2} / \mathrm{AlN}(\mathrm{GaN})$ heterostructures. J Phys Chem C, 2014, 118: 17594-17599

26 Harb M, Cavallo L, Basset J M. Major difference in visible-light photocatalytic features between perfect and self-defective $\mathrm{Ta}_{3} \mathrm{~N}_{5}$ materials: A screened Coulomb hybrid DFT investigation. J Phys Chem C, 2014, 118: 20784-20790

$27 \mathrm{He} \mathrm{C}$, Zhang $\mathrm{J} \mathrm{H}$, Zhang W X, et al. Type-II InSe/g- $\mathrm{C}_{3} \mathrm{~N}_{4}$ heterostructure as a high-efficiency oxygen evolution reaction catalyst for photoelectrochemical water splitting. J Phys Chem Lett, 2019, 10: 3122-3128

28 Modak B, Srinivasu K, Ghosh S K. Photocatalytic activity of $\mathrm{NaTaO}_{3}$ doped with N, Mo, and (N,Mo): A hybrid density functional study. J Phys Chem C, 2014, 118: 10711-10719

29 Shang J, Pan L, Wang X, et al. Tunable electronic and optical properties of InSe/InTe van der Waals heterostructures toward optoelectronic applications. J Mater Chem C, 2018, 6: 7201-7206

30 Zheng Z, Zu X, Zhang Y, et al. Rational design of type-II nano-heterojunctions for nanoscale optoelectronics. Mater Today Phys, 2020, 15: 100262

31 Xia C, Du J, Xiong W, et al. A type-II GeSe/SnS heterobilayer with a suitable direct gap, superior optical absorption and broad spectrum for photovoltaic applications. J Mater Chem A, 2017, 5: 13400-13410

32 Almadori Y, Bendiab N, Grévin B. Multimodal Kelvin probe force microscopy investigations of a photovoltaic $\mathrm{WSe}_{2} / \mathrm{MoS}_{2}$ type-II interface. ACS Appl Mater Interfaces, 2018, 10: 1363-1373

33 Ma Z, Wang S, Li C, et al. Strain engineering for $\mathrm{C}_{2} \mathrm{~N} /$ Janus monochalcogenides van der Waals heterostructures: Potential applications for photocatalytic water splitting. Appl Surf Sci, 2021, 536: 147845

34 Chen Y, Sun H, Peng W. 2D transition metal dichalcogenides and graphene-based ternary composites for photocatalytic hydrogen evolution and pollutants degradation. Nanomaterials, 2017, 7: 62

35 Andrew R C, Mapasha R E, Ukpong A M, et al. Mechanical properties of graphene and boronitrene. Phys Rev B, 2012, 85: 125428

36 Lee C, Wei X, Kysar J W, et al. Measurement of the elastic properties and intrinsic strength of monolayer graphene. Science, 2008, 321: 385-388

37 Zhang S, Zhou J, Wang Q, et al. Penta-graphene: A new carbon allotrope. Proc Natl Acad Sci USA, 2015, 112: 2372-2377

38 Tsafack T, Yakobson B I. Thermomechanical analysis of two-dimensional boron monolayers. Phys Rev B, 2016, 93 : 165434

$39 \mathrm{He} \mathrm{H}$, Lin J, Fu W, et al. $\mathrm{MoS}_{2} / \mathrm{TiO}_{2}$ edge-on heterostructure for efficient photocatalytic hydrogen evolution. Adv Energy Mater, 2016, 6: 1600464

40 Zhang L, Yin J, Wei K, et al. Fabrication of hierarchical $\mathrm{SrTiO}_{3} @ \mathrm{MoS}_{2}$ heterostructure nanofibers as efficient and low-cost electrocatalysts for hydrogen-evolution reactions. Nanotechnology, 2020, 31: 205604

41 Muthurasu A, Maruthapandian V, Kim H Y. Metal-organic framework derived $\mathrm{Co}_{3} \mathrm{O}_{4} / \mathrm{MoS}_{2}$ heterostructure for efficient bifunctional electrocatalysts for oxygen evolution reaction and hydrogen evolution reaction. Appl Catal B-Environ, 2019, 248: 202-210

42 Houssa M, Iordanidou K, Dabral A, et al. Contact resistance at graphene/MoS 2 lateral heterostructures. Appl Phys Lett, 2019, 114: 163101

43 Zhang X, Zhu T, Huang J, et al. Electric field tuning of interlayer coupling in noncentrosymmetric $3 \mathrm{R}-\mathrm{MoS}_{2}$ with an electric double layer interface. ACS Appl Mater Interfaces, 2020, 12: 46900-46907

44 Huang $\mathrm{Z} \mathrm{P}$, Ma B, Wang H, et al. In situ growth of $3 \mathrm{D} / 2 \mathrm{D}\left(\mathrm{CsPbBr}_{3} / \mathrm{CsPb}_{2} \mathrm{Br}_{5}\right)$ perovskite heterojunctions toward optoelectronic devices. J Phys Chem Lett, 2020, 11: 6007-6015

45 Bellus M Z, Li M, Lane S D, et al. Type-I van der Waals heterostructure formed by $\mathrm{MoS}_{2}$ and $\mathrm{ReS}_{2}$ monolayers. Nanoscale Horiz, 2017, 2: 31-36 
46 Luo Y, Wang S, Shu H, et al. A MoSSe/blue phosphorene vdW heterostructure with energy conversion efficiency of $19.9 \%$ for photocatalytic water splitting. Semicond Sci Technol, 2020, 35: 125008

47 Phuc H V, Hieu N N, Hoi B D, et al. Tuning the electronic properties, effective mass and carrier mobility of MoS 2 monolayer by strain engineering: First-principle calculations. J Electron Mater, 2018, 47: 730-736

48 Li M O, Esseni D, Snider G, et al. Single particle transport in two-dimensional heterojunction interlayer tunneling field effect transistor. J Appl Phys, 2014, 115: 074508

49 Nguyen T T, Patel M, Kim S, et al. Transparent photovoltaic cells and self-powered photodetectors by $\mathrm{TiO}_{2} / \mathrm{NiO}$ heterojunction. J Power Sources, 2021, 481: 228865

50 Xie M, Cai B, Meng Z, et al. Two-dimensional BAs/InTe: A promising tandem solar cell with high power conversion efficiency. ACS Appl Mater Interfaces, 2020, 12: 6074-6081

51 Kurtz S R, Myers D, Townsend T, et al. Outdoor rating conditions for photovoltaic modules and systems. Sol Energy Mater Sol Cells, 2000, 62: 379-391

52 Bernardi M, Palummo M, Grossman J C. Semiconducting monolayer materials as a tunable platform for excitonic solar cells. ACS Nano, 2012, 6: 10082-10089

53 Zhou L J, Zhang Y F, Wu L M. SiC 2 siligraphene and nanotubes: Novel donor materials in excitonic solar cells. Nano Lett, 2013, 13: 5431-5436

54 Hou Y, Chen X, Yang S, et al. A band-edge potential gradient heterostructure to enhance electron extraction efficiency of the electron transport layer in high-performance perovskite solar cells. Adv Funct Mater, 2017, 27: 1700878

55 Wu H Y, Yang K, Si Y, et al. Two-dimensional GaX/SnS ${ }_{2}(\mathrm{X}=\mathrm{S}, \mathrm{Se})$ van der Waals heterostructures for photovoltaic application: Heteroatom doping strategy to boost power conversion efficiency. Phys Status Solidi Rapid Res Lett, 2019, 13: 1800565

56 Heo S, Seo G, Cho K T, et al. Dimensionally engineered perovskite heterostructure for photovoltaic and optoelectronic applications. Adv Energy Mater, 2019, 9: 1902470

57 Dou W, Huang A, Ji Y, et al. Strain-enhanced power conversion efficiency of a BP/SnSe van der Waals heterostructure. Phys Chem Chem Phys, 2020, 22: 14787-14795

\section{补充材料}

图S1 在 $d_{0}-1.6$ 和 $d_{0}+1.6 \AA$ 的层间距下, 3 个 $\mathrm{MoS}_{2}-\mathrm{XS}$ 复合结构的能带投影图

图S2 在 -0.1 和 $0.1 \mathrm{~V} / \AA$ 的电场下, 3 个 $\mathrm{MoS}_{2}-\mathrm{XS}$ 复合结构的能带投影图

图 S3 在-4\%和 $4 \%$ 的应变下, 3 个 $\mathrm{MoS}_{2}-\mathrm{XS}$ 复合结构的能带投影图

表S1 $\mathrm{MoS}_{2}-\mathrm{XS}$ 复合结构的晶格参数

本文以上补充材料见网络版csb.scichina.com. 补充材料为作者提供的原始数据, 作者对其学术质量和内容负责. 


\title{
Efficient utilization of light energy based on $\mathrm{MoS}_{2}-\mathrm{XS}(\mathrm{X}=\mathrm{Al}, \mathrm{B}$, Ga) heterostructures
}

\author{
Honglin $\mathrm{Li}^{*}$, Yuanqiang Xiong, Hong Zhang, Lijuan Ye \& Wanjun $\mathrm{Li}^{*}$ \\ Chongqing Key Laboratory of Photo-Electric Functional Materials, College of Physics and Electronic Engineering, Chongqing Normal University, \\ Chongqing 401331, China \\ * Corresponding authors, E-mail: 20170025@cqnu.edu.cn; liwj@cqnu.edu.cn
}

In recent years, two-dimensional transition metal sulfide materials have received widespread attention due to their unique physical and chemical properties, and have been successfully applied in many fields such as microelectronic devices, photovoltaic technology and hydrogen evolution catalysis. However, high recombination rate, low mobility, and low cycle stability all limit the further improvement of the related properties of this kind of material to varying degrees. In our work, two-dimensional materials are composited to optimize the band structure and physical/chemical properties. This kind of approach has been proved to be feasible in many experiments. Not long ago, Demirci et al. studied the III-VI twodimensional single-layer hexagonal structure $\mathrm{MX}(\mathrm{M}=\mathrm{B}, \mathrm{Al}, \mathrm{Ga}, \mathrm{In} ; \mathrm{X}=\mathrm{O}, \mathrm{S}, \mathrm{Se}, \mathrm{Te})$. A series of analyses show that all the above binary compounds are structurally stable. They believe that the III-VI group of binary monolayer materials can be widely used for high-performance optoelectronic devices. Based on density functional theory, this work proposes a new heterostructure form of $\mathrm{MoS}_{2}-\mathrm{XS}(\mathrm{X}=\mathrm{Al}, \mathrm{B}, \mathrm{Ga})$. Both $\mathrm{MoS}_{2}$ and XS $(\mathrm{M}=\mathrm{B}, \mathrm{Al}, \mathrm{Ga})$ have the same hexagonal crystal structure $(\mathrm{P}-6 \mathrm{~m} 2)$, and there are reasons to believe that the $\mathrm{MoS}_{2}$-XS heterogeneous structures will be fabricated under certain experimental condition. Therefore, it is meaningful to understand how the single-layer $\mathrm{MoS}_{2}$ and XS form a stable two-dimensional heterostructure. At present, $\mathrm{MoS}_{2}$-XS based structural characteristics and related physical/chemical properties of the heterostructures have not been reported. Therefore, the purpose of this research is to grasp the basic characteristics and potential application fields of the $\mathrm{MoS}_{2}-\mathrm{XS}$ as far as possible. Based on the periodic boundary conditions of the two-dimensional structure, the establishment of a heterostructure requires us to strictly control the mismatch between $\mathrm{MoS}_{2}$ and XS. We have obtained three highly reliable heterostructures, which maintain the lowest binding energies as well as the smallest mismatches after a series of preliminary calculations. The VBM and CBM of four pristine materials are composed of S atomic states to varying degrees, especially $\mathrm{MoS}_{2}$ and AlS. Almost all of their band edges are derived from $\mathrm{S}$ atoms, which will greatly enhance the recombination of carriers. Therefore, the electrons and holes that can be actually used in the reaction are significantly reduced. How to suppress the recombination of carriers will be one of main goals in this work. The electronic properties of $\mathrm{MoS}_{2}$-XS will be significantly affected by external strain. We conducted a detailed analysis and discussion on how the strain affects the band gap and band edges. From variable interlayer spacing, electric field to strain, $\mathrm{MoS}_{2}$-AlS exhibits good catalytic activity, and has an ideal type-II band alignment. Therefore, we believe that $\mathrm{MoS}_{2}$-AlS is the most ideal choice in terms of photocatalytic water splitting. It is proved that $\mathrm{MoS}_{2}$-BS is unsuitable for the application of water splitting, but it has excellent efficiency in solar cell applications and its PCE can be as high as $\sim 20.4 \%$. It is worth noting that this value is very close to or even better than that of the reported heterostructure solar cells. In short, we believe that this work provides a meaningful reference for how to efficiently use light energy in two-dimensional composite materials.

$\mathrm{MoS}_{2}$, 2D materials, III-VI compounds, heterojunction, first-principles calculations

doi: 10.1360/TB-2021-0307 